\title{
IAMJ
}

INTERNATIONAL

AYURVEDIC

MEDICAL JOURNAL

Review Article

ISSN: 2320-5091

Impact Factor: 6.719

\section{VAJRA KANJIKA - ITS BENEFITS ON SUTIKA}

\section{Rajani Kagga}

Assistant Professor, Department of Prasuti Tantra \& Stree Roga

Sri Sri College of Ayuvedic Science \& Research Centre, Bangalore - 560082, Karnataka, India

Corresponding Author: drcrajanii@gmail.com

https://doi.org/10.46607/iamj2609072021

(Published Online: July 2021)

Open Access

(C) International Ayurvedic Medical Journal, India 2021

Article Received: 25/06//2021 - Peer Reviewed: 26/06/2021 - Accepted for Publication: 27/06/2021

\section{Check for updates}

\section{ABSTRACT}

A woman immediately after giving birth to a child is called Sutika. Various Acharya's have mentioned Sutika Kala as a very important period because the mother is prone to many complications following the delivery, where a lot of Rakta and Kleda are lost in the process of delivery leaving her vulnerable for various disorders. As we know Vata fills the Rikta Sthana and causes various disorders, in a similar way Sutika is very much prone to Vata disorders. Mainly we come across disorders like inadequacy of breast milk and Sutika Makkala (pain). Sthanya is very important in the growth and development of the newborn. It enhances the immunity of the child. Breast milk is babies specific hence one should adopt measures to improve lactation instead of depending on supplementary feeds. Another important condition is Sutika Makkala which is caused due to vitiation of Vata, and shows symptoms like severe discomfort, pain below the umbilical and bladder region, abdomen and cardiac region. At times the pain is so severe that a prickly sensation with a possibility of tearing of bowel or its perforation. These are associated with flatulence and retention of urine. In these above conditions, Vajra Kanjika is of utmost benefit. We get the reference of Vajrakanjika in Yogaratnakara in Ksheera Dosha Chikitsa Adhyaya. Vajra Kanjika contains the drugs like Pippali, Pippali mula, Chavya, Shunti, Yawanika, both Jeeraka's, both Haridra's, Vida and Souvarchala Lavana. It is indicated in Amavata, it is Vrishya, suppresses Kapha and Vata, increases appetite, cures Makkala Shula and increases the quantity of milk. 
In this article, an attempt is made to understand the probable mode of action of Vajra Kanjika in Sutika, Sutika Makkala and Sthanya kshaya.

Keywords: Vajra Kanjika, Sutika, Sutika Roga, Makkala, Sthanya Dosha.

\section{INTRODUCTION}

Ayurveda always emphasizes two aspects Swathasya Swasthya Rakshanam that is protecting the health of a healthy individual so that they would not fall prey to diseases along with maintaining good health and Aaturasya Vikara Prashamanam that is if unhealthy curing the disease and restoring the health of the individual ${ }^{1}$. But mainly it aims to maintain the health in all conditions, for the same reason it has various Paricharyas like Dinacharya, Ratricharya, Rutucharya. Considering the physiological changes, vulnerability and phases the woman goes through at different phases of life Acharya's have mentioned special Paricharyas for women like Rajaswala Paricharya, Garbhadana Samskara, Garbhini Paricharya and Sutika Paricharya to maintain the health of the women during those physiological changes. Acharya Kashyapa says that mothers one leg is in Ihaloka and another leg is in Yamaloka stating the vulnerability of the woman who is about to deliver the child hence utmost care to be taken during and after the delivery ${ }^{2}$. Those who have followed proper Garbhini Paricharya will not face any difficulties during labor ${ }^{3}$. A woman who delivered recently is called Sutika and for her Sutika paricharya is mentioned to restore the optimal health, failing which she is prone to various Sutika Rogas which in turn hamper the health and also hamper the growth and development of the newborn. The women who had difficulties in labour are also prone to various Sutika Roga ${ }^{4}$.

\section{AIM AND OBJECTIVE}

1. To review the literature mentioned in various Ayurveda Samhita's regarding Vajra kanjika

2. To show the clinical significance of Vajra Kanjika in Sutika, Sutika Roga

\section{MATERIALS AND METHODS}

Material: Relevant literature is referred in Samhita's, Samgraha Granthas and Contemporary literature

Methodology: Review study

\section{Sutika \& Sutika kala}

After delivering the Garbha and Apara the woman is considered as Sutika ${ }^{5}$. It's a period during which the delivered mother returns to her pre-pregnant stage. Various physiological changes occur during this time to recuperate the woman and restore her health which was altered during the process of labour.

Table 1:

\begin{tabular}{|c|c|}
\hline Samhita & Duration \\
\hline Sushruta Samhita & $11 / 2$ months $^{6}$ \\
\hline Asthanga Samgraha & $1 \frac{1}{2}$ months $^{7}$ \\
\hline Asthanga Hridaya & $11 / 2$ month, Punarartava Darshana ${ }^{8}$ \\
\hline Kashyapa Samhita & 6 months $^{9}$ \\
\hline Bhava Prakasha & $11 / 2$ months/4 months /Punarartava Darshana ${ }^{10}$ \\
\hline
\end{tabular}

\section{Physiological changes \& Benefits of Sutika Pa- richarya}

These are the following changes that occur in the delivered woman.

\section{Immediately after delivery}

1. During Garbha Vriddhi there is Kshapita and Shithilata of Sarva Sharira Dhatu making her weaker and more tired.

2. Pravahana Vedana - due to bearing down efforts and the process of labour the woman had gone through various pain stimuli. 
3. Kelda Rakta Nisruti-there is the expulsion of various body fluids and blood.

4. Vishesha Shunya Shareera -because of the above condition she becomes exhausted and filled with a feeling of emptiness, in such empty spaces Vata will take advantage and tries to fill those spaces making them vulnerable for various Vata Vyadhi's, Avarana related disorders ${ }^{11}$.

\section{Later phase after delivery}

Just as the Ahara Rasa in Garbhini nourishes Garbha, Sthana, Mother, likewise after the delivery the Ahara Rasa reaches the breasts forming Breast milk and the remaining Ahara Rasa is transformed to blood circulating the whole-body including Yoni and later when the Yoni is nourished and then blood gets accumulated and discharged periodically in the form of menstruation which is mentioned as Punarartava Darshana ${ }^{12}$. Once there is the onset of menstruation it completes the Sutika period, and the normal activities can be resumed. The above said physiological changes are well tackled and normal health is restored only by following various regimens told under Sutika Paricharya. These are the benefits of following proper Sutika Paricharya - the above-mentioned conditions will be corrected, and the woman becomes Punarnavi Bha$v_{a t i}{ }^{13}$ that is proper nourishment and restoration of her optimal health.
Various regimens have been told during this period in terms of Pathya Ahara that is food and nutrition according to Desha, Kala, Jati, Satmya, gender of the born child, personal hygiene lifestyle, improving the psychological condition, Raksha Karma, taking care of a newborn, promoting lactation. Failing to follow the proper regimen the Sutika will be prone to various disorders.

\section{Sutika Roga}

Acharya Kashyapa mentioned 74 Sutika Roga (Sutikopakramaniya Adhyaya 64 and Dushprajata Chikitsa Adhyaya 35) $)^{14,15}$ that afflict the Sutika who fails to follow the proper regimen. A few of these diseases like Shula, Visuchika, Makkala, Sthana Roga hampering the process of lactation can be seen as an immediate squeal and some diseases like Yoni Dosha, Vata Vyadhi etc. can be seen as a late squeal.

As Vata is the main factor which is responsible for causing various disorders Vatahara line of treatment should be adopted. Vajra Kanjika is mentioned by various authors for its efficacy on Sutika related disorders. Vajra kanjika ${ }^{16,17,18 \text {, and } 19}$ - it is herbal preparation containing the following ingredients listed in Table: 2, its Rasa Panchaka in Table: 3, its Phala Shruti in Table: 4 according to various Acharya's respectively.

\section{Table 2: INGREDIENTS}

\begin{tabular}{|l|l|l|l|l|}
\hline & Yoga Ratnakara & Bhaishajya Ratnavali & Chakradatta & Basava Rajeeyam \\
\hline Pippali & + & + & + & + \\
\hline Pippali Mulam & + & + & + & + \\
\hline Chavya & + & + & + & + \\
\hline Shunti & + & + & + & + \\
\hline Yawanika & + & + & + & + \\
\hline Jeeraka & + & + & + & + \\
\hline Krishna Jeeraka & + & + & + & + \\
\hline Haridra & + & + & + & + \\
\hline Daruharidra & + & + & + & + \\
\hline Vida Lavana & + & + & + & + \\
\hline Souvarchala Lavana & + & + & + & + \\
\hline Aranala & + & + & + & + \\
\hline
\end{tabular}


Table 3: RASA PANCHAKA OF INGREDIENTS

\begin{tabular}{|c|c|c|c|c|c|c|c|c|c|}
\hline Name & $\begin{array}{l}\text { Botani- } \\
\text { cal Name }\end{array}$ & Family & Rasa & Guna & $\begin{array}{l}\text { Veery } \\
a\end{array}$ & Vipaka & Karma & $\begin{array}{l}\text { Chemical } \\
\text { Composi- } \\
\text { tion }\end{array}$ & $\begin{array}{l}\text { Pharmaco- } \\
\text { logical ac- } \\
\text { tivity }\end{array}$ \\
\hline Pippali $^{20}$ & $\begin{array}{l}\text { Piper } \\
\text { Longum }\end{array}$ & $\begin{array}{l}\text { Pipe- } \\
\text { raceae }\end{array}$ & Katu & $\begin{array}{l}\text { Laghu, } \\
\text { Snigdha, } \\
\text { Tikshna }\end{array}$ & Ushna & $\begin{array}{l}\text { Madhur } \\
a\end{array}$ & $\begin{array}{l}\text { Vata } \\
\text { Shleshma } \\
\text { Hara, Deep- } \\
\text { ana, } \\
\text { Vrishya, Ra- } \\
\text { sayana. }\end{array}$ & $\begin{array}{l}\text { Piperine, } \\
\text { Piper- } \\
\text { amide, } \\
\text { Pipera- } \\
\text { mine, } \\
\text { Pipene. }\end{array}$ & $\begin{array}{l}\text { Antimicro- } \\
\text { bial, Antiox- } \\
\text { idant, Anti- } \\
\text { inflamma- } \\
\text { tory, Hepa- } \\
\text { toprotective, } \\
\text { Digestive, } \\
\text { Immuno- } \\
\text { modulatory, } \\
\text { Analgesic, } \\
\text { Acts On Me- } \\
\text { tabolism, } \\
\text { Antidepres- } \\
\text { sant Activ- } \\
\text { ity, Anti } \\
\text { Spasmodic, } \\
\text { Cholagauge, } \\
\text { To Check } \\
\text { PPH }\end{array}$ \\
\hline $\begin{array}{l}\text { Pippali } \\
\text { Mula }^{21}\end{array}$ & $\begin{array}{l}\text { Piper } \\
\text { Longum }\end{array}$ & $\begin{array}{l}\text { Pipe- } \\
\text { raceae }\end{array}$ & Katu & $\begin{array}{l}\text { Laghu, } \\
\text { Ruksha }\end{array}$ & Ushna & Katu & $\begin{array}{l}\text { Kapha Vata } \\
\text { Hara, } \\
\text { Bhedana, } \\
\text { Deepana } \\
\text { Pachana. }\end{array}$ & $\begin{array}{l}\text { Piperine, } \\
\text { beta-sitos- } \\
\text { terol, es- } \\
\text { sential oil, } \\
\text { cepharadi- } \\
\text { ones. }\end{array}$ & $\begin{array}{l}\text { Antimicro- } \\
\text { bial, Antiox- } \\
\text { idant, Anti- } \\
\text { inflamma- } \\
\text { tory, Hepa- } \\
\text { toprotective, } \\
\text { Digestive, } \\
\text { Immuno- } \\
\text { modulatory, } \\
\text { Analgesic, } \\
\text { Acts On Me- } \\
\text { tabolism, } \\
\text { Antidepres- } \\
\text { sant Activ- } \\
\text { ity, Anti } \\
\text { Spasmodic. }\end{array}$ \\
\hline Chavya $_{2}^{2}$ & $\begin{array}{l}\text { Piper } \\
\text { Chaba }\end{array}$ & $\begin{array}{l}\text { Pipe- } \\
\text { raceae }\end{array}$ & Katu & $\begin{array}{l}\text { Laghu, } \\
\text { Ruksha }\end{array}$ & Ushna & Katu & $\begin{array}{l}\text { Kapha Vata- } \\
\text { hara, Deep- } \\
\text { ana, Pa- } \\
\text { chana. }\end{array}$ & $\begin{array}{l}\text { Piperine, } \\
\text { Piplartine, } \\
\text { retrofrac- } \\
\text { tamide } \\
\text { A,B,C,D. }\end{array}$ & $\begin{array}{l}\text { Muscle re- } \\
\text { laxant, An- } \\
\text { algesic, Acts } \\
\text { On Metabo- } \\
\text { lism. }\end{array}$ \\
\hline Shunti $^{23}$ & $\begin{array}{l}\text { Zingiber } \\
\text { Offici- } \\
\text { nale }\end{array}$ & $\begin{array}{l}\text { Scitami- } \\
\text { nae }\end{array}$ & Katu & $\begin{array}{l}\text { Guru, } \\
\text { Ruksha, } \\
\text { Tikshna }\end{array}$ & Ushna & $\begin{array}{l}\text { Madhur } \\
a\end{array}$ & $\begin{array}{l}\text { Vata Kapha } \\
\text { Hara, Deep- } \\
\text { ana } \\
\text { Bhedana. }\end{array}$ & $\begin{array}{l}\text { Gingerol, } \\
\text { Zingerone, } \\
\text { Zingeber- }\end{array}$ & $\begin{array}{l}\text { Anti-Inflam- } \\
\text { matory, An- } \\
\text { tibacterial, } \\
\text { Antifungal, }\end{array}$ \\
\hline
\end{tabular}




\begin{tabular}{|c|c|c|c|c|c|c|c|c|c|}
\hline & & & & & & & & $\begin{array}{l}\text { enre, Es- } \\
\text { sential } \\
\text { Oils, As- } \\
\text { partic } \\
\text { Acid, } \\
\text { Threonine, } \\
\text { Serine, } \\
\text { Glycine, } \\
\text { Cysteine, } \\
\text { Valine, } \\
\text { Isoleucine, } \\
\text { Leucine, } \\
\text { Arginine. }\end{array}$ & $\begin{array}{l}\text { Analgesic, } \\
\text { Hepatopro- } \\
\text { tective, An- } \\
\text { tioxidant, } \\
\text { Antidepres- } \\
\text { sant, Anti- } \\
\text { pyretic, An- } \\
\text { ti- } \\
\text { sertoninergi } \\
\text { c, Inhibition } \\
\text { Of Prosta- } \\
\text { glandin Re- } \\
\text { lease }\end{array}$ \\
\hline $\begin{array}{l}\text { Ya- } \\
\text { wanika }^{24}\end{array}$ & $\begin{array}{l}\text { Carum } \\
\text { copticum }\end{array}$ & $\begin{array}{l}\text { Umbel- } \\
\text { lifer- } \\
\text { aceae }\end{array}$ & Katu & $\begin{array}{l}\text { Laghu, } \\
\text { Ruksha, } \\
\text { Tikshna }\end{array}$ & Ushna & Katu & $\begin{array}{l}\text { Kapha Vata } \\
\text { Hara, Deep- } \\
\text { ana, Pa- } \\
\text { chana. }\end{array}$ & $\begin{array}{l}\text { Cam- } \\
\text { phene, } \\
\text { Carvacrol, } \\
\text { Thymine, } \\
\text { Thymol. }\end{array}$ & $\begin{array}{l}\text { Antimicro- } \\
\text { bial, Antibi- } \\
\text { otic, Diu- } \\
\text { retic, Anti- } \\
\text { septic, Anti- } \\
\text { cholinergic, } \\
\text { Antifungal, } \\
\text { Stimulant, } \\
\text { Tonic, Anti- } \\
\text { spasmodic, } \\
\text { Carmina- } \\
\text { tive, }\end{array}$ \\
\hline $\begin{array}{l}\text { Jeeraka }{ }^{2} \\
\end{array}$ & $\begin{array}{l}\text { Cum- } \\
\text { inum } \\
\text { Cyminu } \\
\text { m }\end{array}$ & $\begin{array}{l}\text { Umbel- } \\
\text { lifer- } \\
\text { aceae }\end{array}$ & Katu & $\begin{array}{l}\text { Laghu, } \\
\text { Ruksha }\end{array}$ & Ushna & Katu & $\begin{array}{l}\text { Kapha Vata } \\
\text { Hara Deep- } \\
\text { ana Pa- } \\
\text { chana, } \\
\text { Grahi, } \\
\text { Vrishya, } \\
\text { Garbhashay } \\
\text { a Shodhaka, } \\
\text { Balya. }\end{array}$ & $\begin{array}{l}\text { Cuminal, } \\
\text { Safranal, } \\
\text { Mycene, } \\
\text { Cumino- } \\
\text { side, Ami- } \\
\text { noacids, } \\
\text { Phospho- } \\
\text { lipids. }\end{array}$ & $\begin{array}{l}\text { Antimicro- } \\
\text { bial, Anti- } \\
\text { spasmodic, } \\
\text { Stomachic, } \\
\text { Anti-Inflam- } \\
\text { matory, An- } \\
\text { algesic, Car- } \\
\text { minative, } \\
\text { Antiseptic, } \\
\text { Anti-Fun- } \\
\text { gal, Stimu- } \\
\text { lant, Lacta- } \\
\text { gogue. }\end{array}$ \\
\hline $\begin{array}{l}\text { Krishna } \\
\text { Jeeraka }^{2} \\
6\end{array}$ & $\begin{array}{l}\text { Carum } \\
\text { Carvi }\end{array}$ & $\begin{array}{l}\text { Umbel- } \\
\text { lifer- } \\
\text { aceae }\end{array}$ & Katu & Ruksha & Ushna & Katu & $\begin{array}{l}\text { Kapha } \\
\text { Hara, } \\
\text { Medhya, } \\
\text { Grahi, } \\
\text { Garbhashay } \\
\text { a Visho- } \\
\text { dhaka. }\end{array}$ & $\begin{array}{l}\text { Cuminalde } \\
\text { hyde, Car- } \\
\text { vone, } \\
\text { Menthone, } \\
\text { Carveol, } \\
\text { Limonene, } \\
\text { Abscisic } \\
\text { Acid. }\end{array}$ & $\begin{array}{l}\text { Antispas- } \\
\text { modic, Anti- } \\
\text { bacterial, } \\
\text { Larvicidal, } \\
\text { Antifungal, } \\
\text { Antiseptic, } \\
\text { Stomachic, } \\
\text { Carmina- } \\
\text { tive, Stimu- } \\
\text { lant, Tonic, }\end{array}$ \\
\hline
\end{tabular}




\begin{tabular}{|c|c|c|c|c|c|c|c|c|c|}
\hline & & & & & & & & & $\begin{array}{l}\text { Diuretic, } \\
\text { Reduces } \\
\text { Breast } \\
\text { Swelling. }\end{array}$ \\
\hline$\underset{7}{\text { Haridra }^{2}}$ & $\begin{array}{l}\text { Curcuma } \\
\text { Longa }\end{array}$ & $\begin{array}{l}\text { Zinzeber } \\
\text { aceae } \\
\text { /Scitami } \\
\text { nae }\end{array}$ & $\begin{array}{l}\text { Tikta, } \\
\text { Katu }\end{array}$ & $\begin{array}{l}\text { Ruksha, } \\
\text { Laghu }\end{array}$ & Ushna & Katu & $\begin{array}{l}\text { Kapha, Vata } \\
\text { Hara, Lek- } \\
\text { hana, } \\
\text { Varnya, } \\
\text { Vishaghna. }\end{array}$ & $\begin{array}{l}\text { Curcumi- } \\
\text { noids, } \\
\text { Phytoster- } \\
\text { ols, Fatty } \\
\text { Acids, } \\
\text { Polysac- } \\
\text { charides, } \\
\text { Essential } \\
\text { Oil. }\end{array}$ & $\begin{array}{l}\text { Antibacte- } \\
\text { rial, Chola- } \\
\text { gauge, Anti- } \\
\text { fungal, Anti- } \\
\text { Inflamma- } \\
\text { tory, Anti- } \\
\text { histamic, } \\
\text { Antihepato- } \\
\text { toxic. }\end{array}$ \\
\hline $\begin{array}{l}\text { Daru } \\
\text { Haridra }^{2}\end{array}$ & $\begin{array}{l}\text { Berri- } \\
\text { beris } \\
\text { Aristata }\end{array}$ & $\begin{array}{l}\text { Beriberi- } \\
\text { daceae }\end{array}$ & $\begin{array}{l}\text { Tikta, } \\
\text { Kashaya } \\
m\end{array}$ & $\begin{array}{l}\text { Laghu, } \\
\text { Ruksha }\end{array}$ & Ushna & Katu & $\begin{array}{l}\text { Kapha Pitta } \\
\text { Hara, } \\
\text { Chedana. }\end{array}$ & $\begin{array}{l}\text { Berberine, } \\
\text { Ber- } \\
\text { bamine, } \\
\text { Aromo- } \\
\text { line, Pal- } \\
\text { matine. }\end{array}$ & $\begin{array}{l}\text { Antifatigue, } \\
\text { Antipyretic, } \\
\text { Local Anes- } \\
\text { thetic, Anti- } \\
\text { Inflamma- } \\
\text { tory, Anti- } \\
\text { Bacterial. }\end{array}$ \\
\hline $\begin{array}{l}\text { Vida La- } \\
\text { vana }^{29}\end{array}$ & $\begin{array}{l}\text { Ammo- } \\
\text { nium } \\
\text { Salt }\end{array}$ & & Lavana & $\begin{array}{l}\text { Sakshara } \\
\text { Tik- } \\
\text { shna, } \\
\text { Sukshma } \\
\text {, Vyavayi }\end{array}$ & Ushna & & $\begin{array}{l}\text { Deepana, } \\
\text { Shulahara, } \\
\text { Rochana, } \\
\text { Vatanulo- } \\
\text { mana. }\end{array}$ & Minerals & $\begin{array}{l}\text { Antioxidant } \\
\text { property }\end{array}$ \\
\hline $\begin{array}{l}\text { Sou- } \\
\text { varchala } \\
\text { Lavana }^{30}\end{array}$ & $\begin{array}{l}\text { Sochal } \\
\text { Salt }\end{array}$ & & Lavana & $\begin{array}{l}\text { Laghu, } \\
\text { Sukshma } \\
\text {, Vishada }\end{array}$ & Ushna & & $\begin{array}{l}\text { Shula, } \\
\text { Vibandha } \\
\text { Hara, } \\
\text { Gulma } \\
\text { Hara, Hri- } \\
\text { dya, Udgara } \\
\text { Shuddhi. }\end{array}$ & Minerals & $\begin{array}{l}\text { Antioxidant } \\
\text { property }\end{array}$ \\
\hline
\end{tabular}

Table 4: PHALA SHRUTI BY VARIOUS ACHARYAS:

\begin{tabular}{|l|l|l|l|}
\hline Yogaratnakara & Bhaishajya Ratnavali & Chakradatta & Basava Rajeeyam \\
\hline Amavata Hara & Amadosha Hara & Amavata Hara & Amavata Hara \\
\hline Vrishyam & Vrishyam & Vrishyam & Vrishyam \\
\hline Kaphaghnam & Kaphaghnam & Kaphaghnam & Kaphaghnam \\
\hline Agni Vivardhana & Vahni Deepana & Vahni Deepana, & Agni Deepana \\
\hline Prasuta Sukha Mashnute & - & Streenam Agni Vivardhana & Sutika Roga Shamaka \\
\hline Makkala Shula Shaman & Makkala Shula Shamana & Makkallashula Shamana & Shulaghna \\
\hline Param Ksheera Vardhanam & Param Ksheera Abhivardhanam & Param Ksheera Abhivardhanam & Ksheera Vardhanam \\
\hline Vata Nashanam & - & - & - \\
\hline
\end{tabular}


Table 5: METHOD OF PREPARATION

\begin{tabular}{|l|l|}
\hline Drug & Quantity \\
\hline Pippali & 1 part \\
\hline Pippali mula & 1 part \\
\hline Chavya & 1 part \\
\hline Shunti & 1 part \\
\hline Yawanika & 1 part \\
\hline Jeeraka dvaya & 1 part each \\
\hline Haridradvaya & 1 part each \\
\hline Vida Lavana & 1 part \\
\hline Souvarchala Lavana & 1 part \\
\hline
\end{tabular}

The above-mentioned drugs in Table: 5 with equal quantity are made into a paste and cooked in Aranala ${ }^{31}$ and consumed. It can also be prepared in the form of Ksheerapaka ${ }^{32,33,34}$ and consumed based on Agnibala of Sutika.

\section{DISCUSSION}

During pregnancy there are progressive anatomical and physiological changes not only confined to the genital organs but also to all systems of the body ${ }^{35}$ and during labour woman is exhausted due to bearing down efforts, straining, loss of the body fluids, blood, energy making her more vulnerable to infections. After the birth of the child, all the changes during pregnancy will revert to a pre-pregnant state ${ }^{36}$, hence there are various biochemical and metabolic changes happening in the body and this is the time she is vulnerable and prone to various infections and diseases if not taken adequate care. Also, if the woman had faced difficulties and complications during labour and not followed the regimen properly then she is prone to various Vata related disorders because of Shunya Shareera, Nisruta Rakta Dhatu. Because of Kshapita and Shithila of Shareera there could be Jatharagni Mandhya and Dhatwagni Mandhyata leading to impairment in the metabolism. Agni mandhyata leads to Ama Utpatti, whatever food consumed will not be aiding for nourishment instead will aid in disease pathology. Though the baby is delivered it is still dependent on its mother for its survival, nourishment through lactation. Whatever food consumed by the mother is intended for own nourishment and breast nourishment for proper lactation, but because of imbalance in the
Agni, this process cannot happen normally. In such cases, Vajra Kanjika is very much beneficial in curing and preventing Sutika related diseases. Most of its ingredients are having Ushna Virya, Laghu Ruksha Guna and Kapha Vata Shamana, Vrishya, Deepana, Pachana, Garbhashaya Shodhaka properties hence acting of conditions like Agni Deepana, Ama Dosha, Shula, Sthanya Kshaya and Dushti.

1. Agni Deepana Karma, Ama Pachana: As the body is trying to revert to its pre-pregnant state due to various chemical changes occurring in the body there could be impairment in the metabolic and biochemical process. Most ingredients in Vajra Kanjika having characteristic features like Ushna Virya, Laghu, Ruksha Guna and Kapha Vata Shamana, Deepana, Pachana and their various chemical components have shown properties like Carminative, Stimulant, Digestive, Stomachic, Cholagauge, Hepatoprotective, aids in metabolism helps in normalizing the Agni and eliminating the root cause for various disorders during Sutikavastha.

2. Rakshoghna Karma-Maternal injuries following the childbirth process are quite common and contribute significantly to maternal morbidity and even to death, effective management not only minimize the morbidity but prevent many a gynaecological problem from developing later in life ${ }^{37}$. $\mathrm{Va}$ jra Kanjika contents are having properties like Antiseptic Antibacterial, Antifungal, Antihelminthic, Antipyretic, Anti-inflammatory action will help in preventing and curing the infections also aiding better would healing and boosting the immunity. 
3. Shula Hara, Makkala Shula - This is a condition wherein there is severe pain in the abdomen below umbilicus due to accumulation of collected blood after delivery due to improper usage of cleansing articles. There is a pricking type of pain in Pakwashaya, Basti, and Murdha region causing discomfort to the female ${ }^{38}$. This can be understood as After pain characterized by infrequent, spasmodic pain felt in lower abdomen after delivery for a variable period of 2-4 days and there is the presence of blood clots or bits of the afterbirths leading to hypertonic contractions of the uterus in an attempt to expel the out. Modern practices prescribe Analgesics and Antispasmodics to relieve the pain ${ }^{39}$. Vajra Kanjika helps in controlling Makkala Shula as the drugs are having properties like Lekhana, Bhedana helping in the escape of collected blood and further doing Garbhashaya Shodhana. Also, with the property of Vata hara action, the vitiated Vata is pacified and automatically the Shula is relieved. Also, various chemical components present in this formulation have shown Analgesic, Antispasmodic, Muscle relaxant Anti-inflammatory activity.

4. Sthanya Vardhanam - Due to the formation of Ama and or improper Garbhini Paricharya there might be a problem in Mammogenesis, Lactogenesis and due to difficult labour and improper Sutika Paricharya there is a chance of impairment in Galactokinesis and Galactopoiesis causing Sthanya Kshaya or Dushti. Breast milk is baby specific; hence one should adopt the measures to enhance proper lactation. The above-mentioned drug is having properties like Amahara, Agni Vardhana helping proper assimilation and metabolism further helping in the proper formation of Rasa Dhatu leading to adequate Ksheera Pravrutti, also by its Lactagogue property found in its various chemical components.

5. Vrishya - During Sutikavastha all the attention will be directed to the baby and the mother feels left out, tiered, less cared and also though all the systems are reverting to pre-pregnant state, her shape and tonicity of the body take more time to revert back or sometimes it might not revert making the mother prone for psychological illness. $\mathrm{Va}$ jra Kanjika contains drugs having Antioxidant, Immunomodulatory, Anti-fatigue, Antidepressant, CNS stimulant, a tonic property which helps in the overall well-being of the mother and also preventing from the affliction of various psychological disorders like Post-partum Blues and Depression.

\section{CONCLUSION}

Vajra Kanjika as the name suggests helps in clearing all the adamant or stubborn conditions causing disturbance to a Sutika. It is beneficial in various Sutika Rogas. Especially it clears the Ama, obstruction to the channels are relieved further promoting normal transportation of Ahara rasa which later nourishes the Sarva Shareera Dhatu. Based on its Guna, Karma and pharmacological studies it is analyzed that Vajra Kanjika helps Sutika in combating physical ailments like Agni Mandhya, Ajeerna, Makkala Shula etc., preventing psychological ailments like Unmada etc., post-partum blues and depression and helping the Newborn by providing adequate lactation. Overall aiding the process of Punarnavikarana of the mother by promoting and restoring optimal health. There is a scope for further research regarding this formulation to understand its clinical application and efficacy.

\section{REFERENCES}

1. Agnivesha, Charaka, Dridhabala, Chakrapani, Arthedasha mahamuleeya adhyaya, Trikamji Y, Varanasi, Chaukhambha Surabharati Prakashan: 2013; P.287

2. Jivaka, Vatsya, Hemaraja, Sutikopakraminya Adhyaya, Kashyapa samhita, Bhishagacharya S, Varanasi, Chaukhambha Sanskrit sansthan;2006, P.304

3. Hemalatha kapoorchand, Garbhini Vignanam, A comprehensive treatise on Prasuti Tantra; Varanasi, Chaukhambha vishwabharati 2019:P.800-805

4. Hemalatha kapoorchand, Sutika roga, A comprehensive treatise on Prasuti Tantra; Varanasi, Chaukhambha vishwabharati 2019:P.800

5. Jivaka, Vatsya, Hemaraja, Sutikopakraminya Adhyaya, Kashyapa samhita, Bhishagacharya S, Varanasi, Chaukhambha Sanskrit sansthan;2006, P.305 
6. Sushruta, Dalhana, Garbhini vyakarana shareeram, Sushruta samhita, Trikamji J, Varanasi, Chaukhambha Subharati Prakashan: 2008; P.389

7. Vagbhata, Arunadatta, Hemadri, Garbhopacharaneeyo adhyaya, Sharma S, Ashtanga Samgraha, $3^{\text {rd }}$ edition: Varanasi, Chaukhambha Sanskrit series office, 2012; P.291

8. Vagbhata, Arunadatta, Hemadri, Garbhavakranti shareeram, Paradakar BH, Ashtanga Hridaya, edition: Varanasi, Chaukhambha Surbharati Prakashan, 2007; P.377

9. Jivaka, Vatsya, Hemaraja, Sutikopakraminya Adhyaya, Kashyapa samhita, Bhishagacharya S, Varanasi, Chaukhambha Sanskrit sansthan;2006, P.308

10. Mishra S, Balaprakaransam, Bhavaprakasha, Vol.1 Varanasi, Chaukhambha samskruta bhavan; 2007, P.96

11. Vagbhata, Arunadatta, Hemadri, Garbhopacharaneeyo adhyaya, Sharma S, Ashtanga Samgraha,3rd edition: Varanasi, Chaukhambha Sanskrit series office, 2012; P.290

12. Jivaka, Vatsya, Hemaraja, Raktagulmachikitsa Adhyaya, Kashyapa samhita, Bhishagacharya S, Varanasi, Chaukhambha Sanskrit sansthan;2006, P.287

13. Vagbhata, Arunadatta, Hemadri, Garbhopacharaneeyo adhyaya, Sharma S, Ashtanga Samgraha,3rd edition: Varanasi, Chaukhambha Sanskrit series office, 2012; P.290

14. Jivaka, Vatsya, Hemaraja, Sutikopakraminya Adhyaya, Kashyapa samhita, Bhishagacharya S, Varanasi, Chaukhambha Sanskrit sansthan;2006, P.305

15. Jivaka, Vatsya, Hemaraja, Dushprajata chikitstita Adhyaya, Kashyapa samhita, Bhishagacharya S, Varanasi, Chaukhambha Sanskrit sansthan;2006, P.97

16. Joshi S.S, Ksheeradosha chikitsa adhyaya, Yogaratnakara, Varanasi, Chaukhambha samskruta pustakalaya; 1939, P.752

17. Lochan K, Sutikaroga chikitsa prakaranam, Bhaishajya Ratnavali, $1^{\text {st }}$ edition, Vol 3, Varanasi, Chaukhambha Sanskrit bhavan; P-401

18. Sharma P.V, Stree roga chikitsa, Chakradatta, Varanasi, chaukhambha orientalia: 2007; P.541

19. Shribasavararaja, Jnanendrapandey, Shodhasha prakaranam, Basavarajiyam, Varanasi, and Chaukhambha krishnadas Academy: 2010; P.499-500

20. Sharma P.C, Yelne M.B, Dennis T.J, Database of Indian medicinal plants VOL 3, New Delhi, Central Council for Research in Ayurveda and Siddha:2001; P. 472-476
21. Shastry J.L.N, Dravyaguna Vijnana, VOL 2, Varanasi, Chaukhambha orientalia:2004; P.561-563

22. Shastry J.L.N, Dravyaguna Vijnana, VOL 2, Varanasi, Chaukhambha orientalia:2004; P.574-575

23. Sharma P.C, Yelne M.B, Dennis T.J, Database of Indian medicinal plants VOL 5, New Delhi, Central Council for Research in Ayurveda and Siddha:2002; P. 315-320

24. Sharma P.C, Yelne M.B, Dennis T.J, Database of Indian medicinal plants VOL 7, New Delhi, Central Council for Research in Ayurveda and Siddha:2005; P. 496-499

25. Sharma P.C, Yelne M.B, Dennis T.J, Database of Indian medicinal plants VOL 8, New Delhi, Central Council for Research in Ayurveda and Siddha:2007; P. 419-426

26. Sharma P.C, Yelne M.B, Dennis T.J, Database of Indian medicinal plants VOL 6, New Delhi, Central Council For Research in Ayurveda and Siddha:200?; P. 86-89

27. Sharma P.C, Yelne M.B, Dennis T.J, Database of Indian medicinal plants VOL 1, New Delhi, Central Council for Research in Ayurveda and Siddha:2000; P. $152-155$

28. Sharma P.C, Yelne M.B, Dennis T.J, Database of Indian medicinal plants VOL 1, New Delhi, Central Council For Research in Ayurveda and Siddha:2000; P. 120-122

29. Shastry J.L.N, Dravyaguna Vijnana VOL 3, Varanasi, Chaukhambha orientalia:2011; P. 78-80

30. Shastry J.L.N, Dravyaguna Vijnana VOL 3, Varanasi, Chaukhambha orientalia:2011; P. 78-80

31. Sharangadhara, Asavavidhanam, Tripathy P, Sharangadhara samhita, Varanasi, chaukhambha surabharati Prakashan; P.235

32. Sharangadhara, Annapakakriya, Tripathy $P$, Sharangadhara samhita, Varanasi, chaukhambha surabharati Prakashan; P. 167

33. Lochan K, Sutikaroga chikitsa prakaranam, Bhaishajya Ratnavali, 1st edition, Vol 3, Varanasi, Chaukhambha Sanskrit bhavan; P-401

34. Sharma P.V, Stree roga chikitsa, Chakradatta, Varanasi, chaukhambha orientalia: 2007; P.541

35. Dutta. D.C, Hiralal Konar, Physiological changes during Pregnancy, 9th Edition, Textbook of Obstetrics, New Delhi, Jaypee the health sciences publisher:2018; P.42 
36. Dutta. D.C, Hiralal Konar, Normal Pueperum, $9^{\text {th }}$ Edition, Textbook of Obstetrics, New Delhi, Jaypee the health sciences publisher:2018; P.135

37. Dutta. D.C, Hiralal Konar, Injuries to the birth canal, $9^{\text {th }}$ Edition, Textbook of Obstetrics, New Delhi, Jaypee the health sciences publisher:2018; P.397

38. Hemalatha kapoorchand, Sutika roga, A comprehensive treatise on Prasuti Tantra; Varanasi, Chaukhambha vishwabharati 2019:P.800

39. Dutta D.C, Hiralal Konar, Normal Pueperum, $9^{\text {th }}$ Edition, Textbook of Obstetrics, New Delhi, Jaypee the health sciences publisher:2018; P.143

\section{Source of Support: Nil \\ Conflict of Interest: None Declared}

How to cite this URL: Rajani Kagga et al: Vajra Kanjika - It's Benefits on Sutika. International Ayurvedic Medical Journal \{online\} 2021 \{cited July 2021\} Available from: http://www.iamj.in/posts/images/upload/1484_1494.pdf 